\title{
Reconciling Imbalanced Transition State Effects with Nonadiabatic CPET Reactions
}

\author{
Joseph E. Schneider, McKenna K. Goetz, John S. Anderson* \\ Department of Chemistry, University of Chicago, Chicago, Illinois 60637, United States
}

ABSTRACT: Recently there have been several experimental demonstrations of how concerted proton electron transfer (CPET) reaction rates are affected by off-main-diagonal energies, namely the stepwise thermodynamic parameters $\Delta \mathrm{G}_{\mathrm{PT}}^{\circ}$ and $\Delta \mathrm{G}_{\mathrm{ET}}^{\circ}$. Semiclassical structure-activity relationships have been invoked to rationalize these asynchronous linear free energy relationships despite the widely acknowledged importance of quantum effects such as nonadiabaticity and tunneling in CPET reactions. Here we report variable temperature kinetic isotope effect data for the asynchronous reactivity of a terminal Cooxo complex with $\mathrm{C}-\mathrm{H}$ bonds and find evidence of substantial quantum tunneling which is inconsistent with semiclassical models even when including tunneling corrections. This indicates substantial nonadiabatic tunneling in the CPET reactivity of this Co-oxo complex and further motivates the need for a quantum mechanical justification for the influence of $\Delta \mathrm{G}_{\mathrm{PT}}^{\circ}$ and $\Delta \mathrm{G}_{\mathrm{ET}}^{\circ}$ on reactivity. To reconcile this dichotomy, we include $\Delta \mathrm{G}_{\mathrm{PT}}^{\circ}$ and $\Delta \mathrm{G}_{\mathrm{ET}}^{\circ}$ in nonadiabatic models of CPET by having them influence the anharmonicity and depth of the proton potential energy surfaces, which we approximate as Morse potentials. With this model we independently reproduce the dominant trend with $\Delta \mathrm{G}_{\mathrm{PT}}^{\circ}+\Delta \mathrm{G}_{\mathrm{ET}}^{\circ}$ as well as the subtle effect of $\Delta \mathrm{G}_{\mathrm{PT}}^{\circ}-\Delta \mathrm{G}_{\mathrm{ET}}^{\circ}$ (or $\eta$ ) in a nonadiabatic framework. The primary route through which these off-diagonal energies influence rates is through vibronic coupling. Our results reconcile predictions from semiclassical transition state theory with models that treat proton transfer quantum mechanically in CPET reactivity and suggest that similar treatments may be possible for other nonadiabatic processes.

\section{Introduction}

A wide array of chemical processes proceed through the concerted transfer of both a proton and an electron (concerted proton electron transfer, CPET). ${ }^{1-5}$ This has motivated a large body of research into what factors control this reactivity. ${ }^{6-19}$ Typically, reactions with a more favorable free energy of reaction ( $\Delta \mathrm{G}^{\circ}$ СРЕT) have a lower free energy of activation $\left(\Delta \mathrm{G}^{\ddagger} \mathrm{CPET}\right) .{ }^{12,18-22}$ However, there has recently been evidence that this well-established reactivity trend is not always dominant. Notably, the rates of some systems also trend with the thermodynamic energies of off-diagonal elements in a classical CPET square scheme, namely individual proton transfer and electron transfer energies $\left(\Delta \mathrm{G}_{\mathrm{PT}}^{\circ}\right.$ and $\Delta \mathrm{G}_{\mathrm{ET}}^{\circ}$, Figure 1). ${ }^{9,23-28}$ Such asynchronous or imbalanced reactivity trends are well precedented in organic hydrogen atom transfer reactions, ${ }^{29}$ but their relevance in the broader class of CPET reactions has implications for the mechanism and selectivity of many biological and synthetic systems. ${ }^{30,31}$

A central paradox that has recently emerged is how the inclusion of off-diagonal thermodynamic elements, which is rooted in semiclassical transition state theory, can be reconciled with CPET reactions which are widely acknowledged to be nonclassical or nonadiabatic (Figure 1). ${ }^{16,32,33}$ The specific formulations for how $\Delta \mathrm{G}_{\mathrm{PT}}^{\circ}$ and $\Delta \mathrm{G}_{\mathrm{ET}}^{\circ}$ can affect

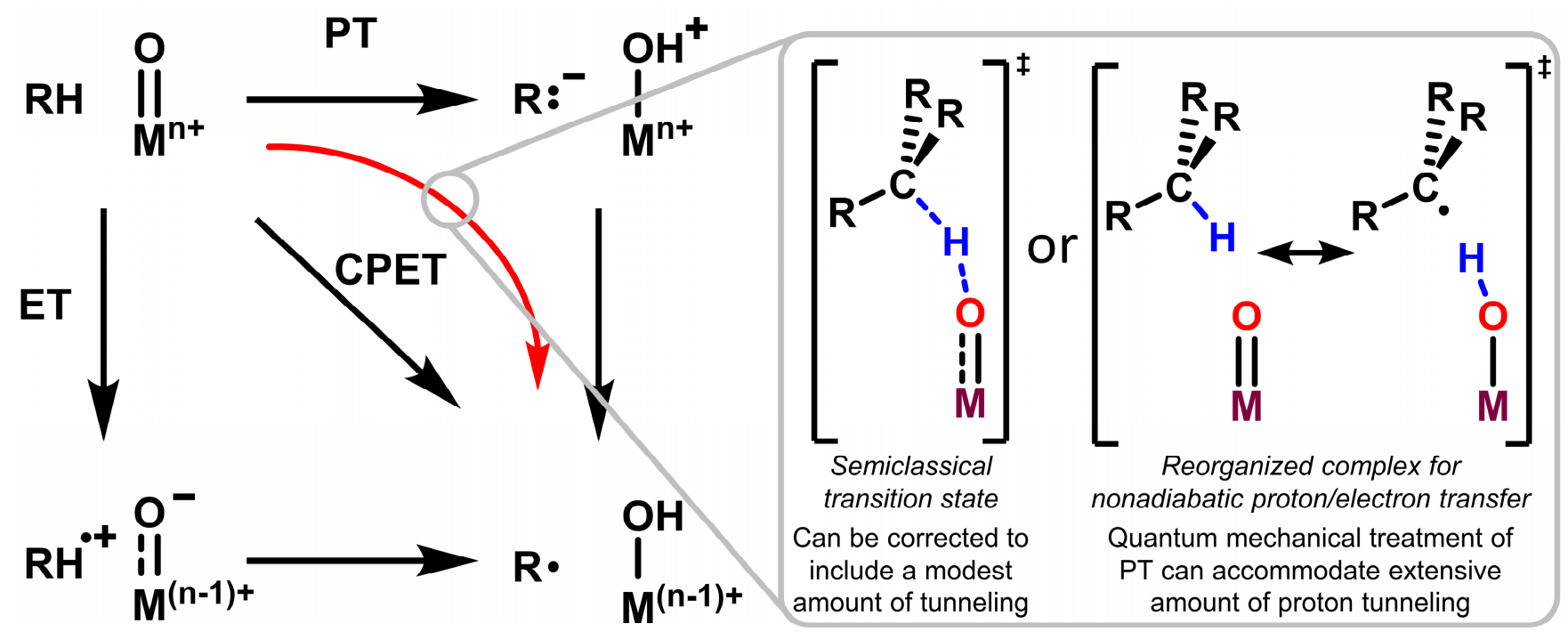

Figure 1. The different pictures for CPET transition states that result from varied methods of accounting for proton tunneling. 
CPET reactivity vary, but are commonly supported by DFT optimized transition states $9,23,26,27$ and invoke textbook physical organic concepts such as nonperfect synchronization, imbalanced transition states, and More O'FerralJencks's diagrams. ${ }^{34-38}$ However, the light mass of protons means that quantum effects such as tunneling are often important, and even dominant, in CPET reactivity. ${ }^{11,39-45}$ While semiclassical transition states can be corrected for a moderate amount of tunneling, ${ }^{39}$ extensive tunneling requires a dedicated quantum mechanical treatment which invokes nonadiabatic proton/electron transfer. ${ }^{40,41,46,47}$ This extensive tunneling precludes the formation of a well-defined transition state with the transferring proton localized halfway between donor and acceptor, as is assumed in semiclassical transition state theory. It is unclear how to reconcile structure-activity relationships from semiclassical transition state theory with the significant quantum effects which can occur in proton transfer, or if such a reconciliation is even feasible. ${ }^{16,23,32,33,48,49}$

Recently our laboratory reported on the reactivity of the terminal Co-oxo complex $\mathrm{PhB}\left({ }^{t} \mathrm{BuIm}\right)_{3} \mathrm{Co}^{\mathrm{III}} \mathrm{O}$ (CoO) with $\mathrm{C}-$ $\mathrm{H}$ bonds (Scheme 1). ${ }^{25}$ It was found that this reaction followed a CPET mechanism, but displayed reactivity trends consistent with a basic asynchronous or imbalanced transition state. Specifically, CoO reacts more quickly with fluorene $\left(\mathrm{p} K_{\mathrm{a}}(\mathrm{DMSO}) 23, \mathrm{BDE} 82 \mathrm{kcal} / \mathrm{mol}\right)$ than it does with 9,10-dihydroanthracene (DHA; $\mathrm{p} K_{\mathrm{a}}$ (DMSO) 30, BDE 76 $\mathrm{kcal} / \mathrm{mol}$ ), which runs counter to traditional $\Delta \mathrm{G}_{\text {СРЕT trends }}^{\circ}$ but aligns with the importance of an imbalanced transition state resulting in a trend with $\Delta \mathrm{G}_{\text {PT }}^{\circ}$ (Scheme 1$) .{ }^{50-55}$ The recent controversy over semiclassical trends in nonadiabatic processes prompted us to experimentally probe the importance of proton tunneling behavior in this system and, if a nonadiabatic treatment is necessary, understand how to reconcile nonadiabatic behavior with the asynchronous trends. ${ }^{16,32,33}$

Here we show that variable temperature kinetic isotope effect (KIE) measurements for the reaction of $\mathbf{C o O}$ with fluorene and DHA show evidence for extensive tunneling most consistent with a nonadiabatic reaction. This result prompted us to evaluate how such a nonadiabatic process could be consistent with the kinetic trends of CoO. We hypothesized that off main-diagonal thermodynamic elements $\Delta \mathrm{G}_{\mathrm{PT}}^{\circ}$ and $\Delta \mathrm{G}_{\mathrm{ET}}^{\circ}$ can directly affect the rates of a nonadiabatic reaction via vibronic coupling. A model built on simple Morse potentials to test this hypothesis rectifies the apparent discrepancy between the quantum mechanical nature of CPET reactions with semiclassical pictures of imbalanced transition states. Furthermore, this model organically predicts trends with off-diagonal parameters that have been observed from both statistical and computational analysis of metal-oxo mediated CPET reactions. Our results show how imbalanced transition state trends can be integrated with nonadiabatic CPET reactions of metal-oxo complexes and demonstrate that semiclassical structure-activity relationships are compatible with quantum mechanical treatments of reactivity.

\section{Results and Discussion}

Variable Temperature KIE Experiments and Implications

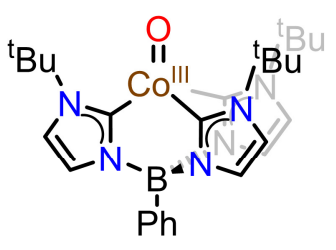

\section{Performs CPET \\ activation of $\mathrm{C}-\mathrm{H}$ \\ bonds with \\ asynchronous \\ reactivity trends}

$\mathrm{PhB}\left({ }^{\mathrm{B}} \mathrm{Bulm}\right){ }_{3} \mathrm{Co}{ }^{\prime \prime \prime O} \mathrm{O}(\mathrm{CoO})$<smiles>c1ccc2c(c1)Cc1ccccc1C2</smiles>

9,10-dihydroanthracene (DHA)

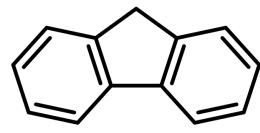

Fluorene

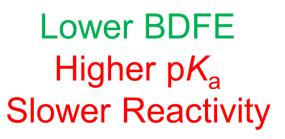

Scheme 1. Compounds discussed in this work.

The importance of tunneling in the proton transfer component of CPET can be probed via the temperature dependence of kinetic isotope effects (KIEs, Figure 3). ${ }^{99,40,56,57}$ The slope of $\ln (\mathrm{KIE})$ vs. $1 / \mathrm{kT}$ gives the difference in activation energy $\left(E_{a}(D)-E_{a}(H)\right)$ between transferring a hydrogen or a deuterium, and the intercept is related to the difference between their exponential prefactors $\left(\ln \left(A_{H} / A_{D}\right)\right)$. In the absence of any tunneling, there are stringent limits on these parameters: $\mathrm{E}_{\mathrm{a}}(\mathrm{D})-\mathrm{E}_{\mathrm{a}}(\mathrm{H})$ must be less than $1.4 \mathrm{kcal} / \mathrm{mol}$, and $\ln \left(A_{H} / A_{D}\right)$ is expected to be approximately zero, with -0.70 and +0.35 being generous bounds (Figure $3 \mathrm{~A}$ ). ${ }^{39,58}$

Tunneling will make a reaction less sensitive to changes in temperature, thereby decreasing the activation energy. This decrease in activation energy is isotope sensitive, with the lighter proton having more efficient tunneling and thereby a sharper drop in $\mathrm{E}_{\mathrm{a}}$ than the same reaction with deuterium. As a result, the difference in activation energies for hydrogen and deuterium increases beyond the 1.4 $\mathrm{kcal} / \mathrm{mol}$ limit assumed with no tunneling, and the extrapolated intercept may be substantially negative (Figure 3B). However, if tunneling is significant enough, then both deuterium and hydrogen will tunnel extensively, leading to similar activation energies and a slope that is once again within the $1.4 \mathrm{kcal} / \mathrm{mol}$ no tunneling limit (Figure 3C). However, in this scenario the intercept does not necessarily need to be zero and can even be significantly positive. This third scenario is difficult to rationalize within semiclassical transition state theory, and the observation of a significantly positive intercept in a $\ln (\mathrm{KIE})$ vs. $1 / \mathrm{kT}$ plot supports modeling proton transfer as nonadiabatic rather than as semiclassical. ${ }^{40,56}$

With these experimental benchmarks in mind, we measured the temperature dependent KIEs of $\mathbf{C o O}$ reacting with both fluorene and DHA (Figure 2, Table 1). We measured KIEs for fluorene oxidation by competition, analyzing the deuteration of the dimerized radical product (bifluorenyl) from reactions with $d_{1}$-fluorene (intramolecular KIEs) and with $50 \% d_{0}$-fluorene/50\% $d_{2}$-fluorene (intermolecular KIEs). This allowed us to determine both the primary and secondary KIEs for this reaction. Kinetic measurements of 


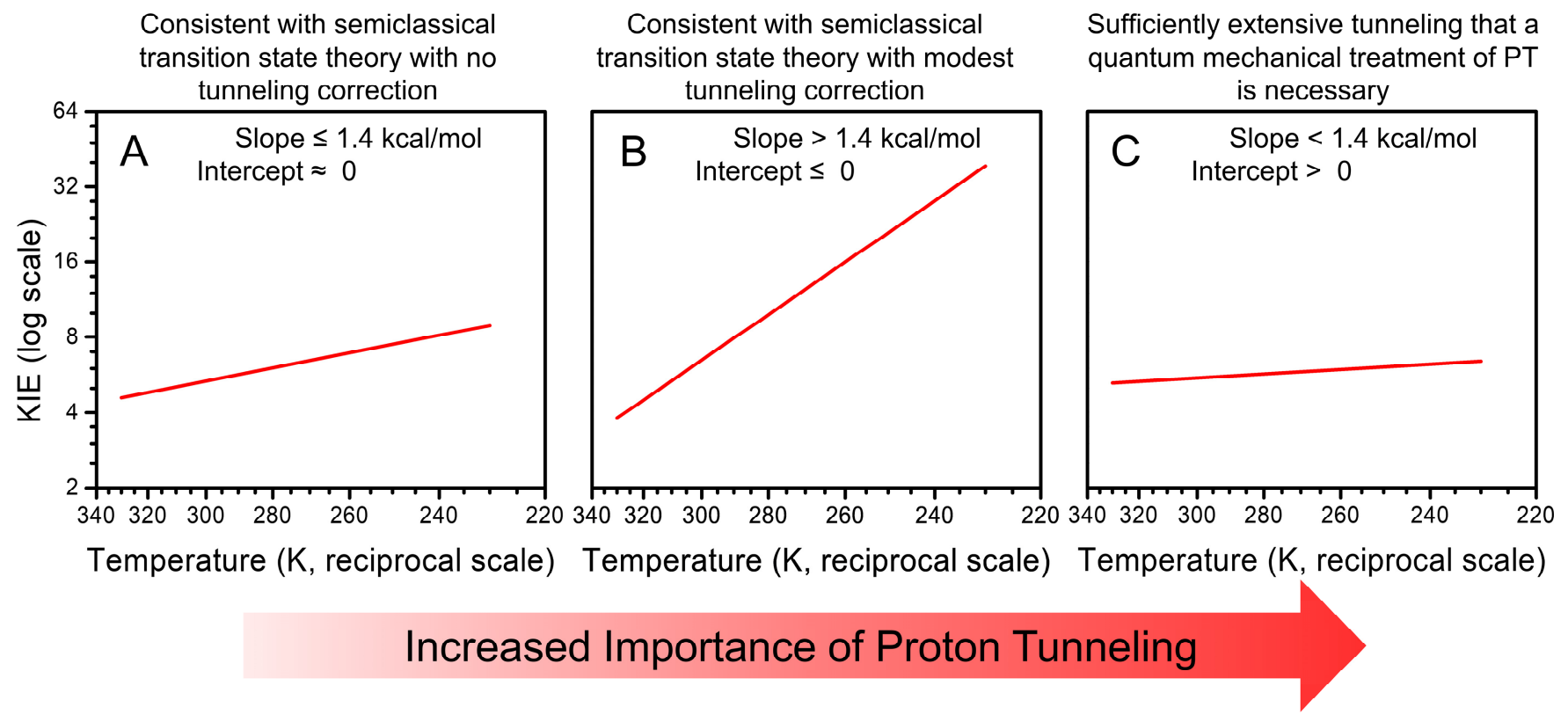

Figure 3. Generic plots of $\ln (\mathrm{KIE})$ vs. 1/kT for systems displaying (A) no proton tunneling, (B) moderate proton tunneling, or (C) extensive proton tunneling. The $\mathrm{x}$-axes are reciprocal scales, and the $\mathrm{y}$-axes are logarithmic scales.

$k_{\mathrm{H}}$ for $\mathrm{d}_{0}$-fluorene and $k_{\mathrm{D}}$ for $\mathrm{d}_{2}$-fluorene gave similar results, but the accuracy was insufficient to confidently assess the intercept (See SI). In contrast, the KIEs from the reaction of CoO with DHA were too large for analysis via product composition; instead, we measured the KIEs by separately measuring $k_{\mathrm{H}}$ and $k_{\mathrm{D}}$ for $d_{0}$-DHA and $d_{4}$-DHA, respectively (See SI).

For fluorene, we observe a shallow slope and significantly positive intercept in the plot of $\ln (\mathrm{KIE})$ vs. $1 / \mathrm{kT}$ which indicates extensive tunneling of both hydrogen and deuterium. This observation supports that tunneling corrections to semiclassical transition states are not sufficient to explain this reactivity, and a more thorough quantum mechanical treatment is necessary; i.e. it corresponds to the scenario in Figure 3C. ${ }^{40,56}$ Specifically, this is evidence for nonadiabatic CPET in which the activation energy is derived not from the partial transfer of the varied isotope but instead from the reorganization of the environment.

Compared to the oxidation of fluorene, the oxidation of DHA has larger KIEs and a steeper slope. This indicates that deuterium tunneling is more sensitive to temperature than proton tunneling for DHA. While these parameters can be reasonably obtained with tunneling corrections to a semiclassical transition state, they are also consistent with a nonadiabatic reaction. For instance, in enzymatic systems such steep slopes are explained within a nonadiabatic framework by invoking deuterium being more sensitive to tunneling distance. ${ }^{56}$ Thus, it is most plausible that nonadiabatic effects are consistently important for all CPET reactions of CoO.

The temperature insensitive KIEs for reactions between CoO and fluorene and the resulting positive value of $\ln \left(A_{H} / A_{D}\right)$ are uncommon in small molecule reactions; however, it is not unprecedented and, in fact, is the norm in enzymatic systems (see Table 1 for a representative sample of other systems). ${ }^{28,59-78}$ Within the more specific context of metal complex mediated $\mathrm{C}-\mathrm{H}$ activation, the paucity of data makes it difficult to draw general conclusions regarding the temperature dependence of KIEs and the importance of tunneling. Most reported data, such as the decomposition of a dicobalt $\mu$-peroxo complex 65 and the oxidation of ethylbenzene by an $\mathrm{Fe}(\mathrm{V})$ oxo complex, ${ }^{64}$ have negative $\ln \left(\mathrm{A}_{\mathrm{H}} / \mathrm{A}_{\mathrm{D}}\right)$ values which are consistent with tunneling but do not necessarily require the nonadiabatic extreme.

Our dichotomous results are most reminiscent of a series of three copper hydroxide complexes' reactivity with DHA. ${ }^{68}$ Two of these complexes display negative $\ln \left(A_{H} / A_{D}\right)$ but one displays positive $\ln \left(\mathrm{A}_{\mathrm{H}} / \mathrm{A}_{\mathrm{D}}\right)$. Similar results have been reported for bis- $\mu$-oxo complexes of copper. ${ }^{63,66,67}$ It is not immediately obvious why reactivity between $\mathbf{C o O}$ and

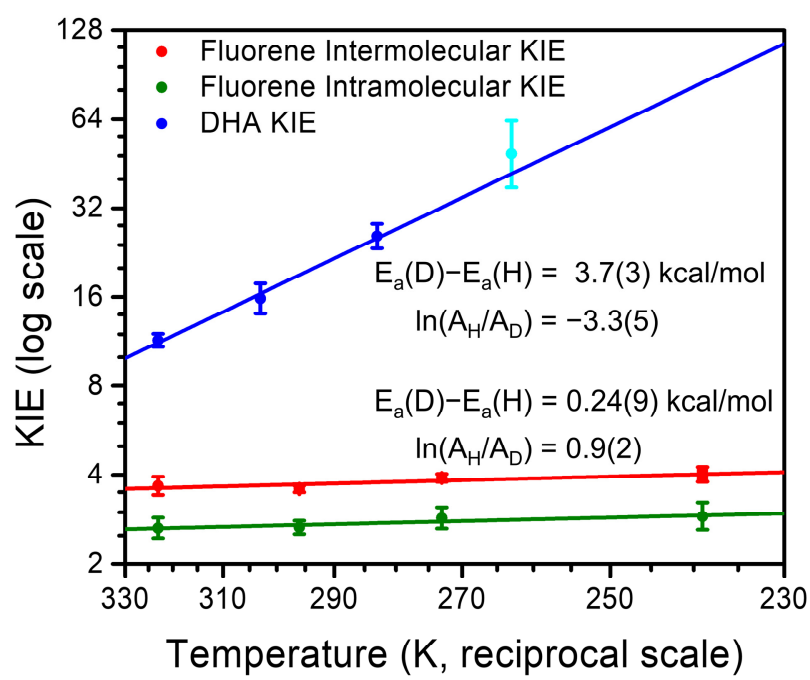

Figure 2. Plots of $\ln (\mathrm{KIE})$ vs $1 / \mathrm{kT}$ for the reactions of $\mathbf{C o O}$ with fluorene and DHA and the corresponding linear fits. The cyan colored data point at $263 \mathrm{~K}$ for DHA was determined via initial rates. This data point is graphed to demonstrate consistency with the other data points, but not included in the linear fits to variable temperature. The $\mathrm{x}$-axis is reciprocal scale, and the $y$-axis is logarithmic scale. 
fluorene and some of these copper reactions are more "enzyme-like" with a positive $\ln \left(\mathrm{A}_{\mathrm{H}} / \mathrm{AD}_{\mathrm{D}}\right)$ whereas reactivity between $\mathbf{C o O}$ and DHA and most small molecule CPET reactions are not; however, these studies suggest that evidence for nonadiabaticity is perhaps best evaluated in a series of reactions rather than with just one substrate.

The possibility of nonadiabatic effects in $\mathrm{C}-\mathrm{H}$ activation has been underappreciated to date. Many hypotheses regarding the enhancement of CPET reactivity in transition metal-oxo complexes have been based solely from DFTcalculated transition states, without consideration of whether alternative, nonadiabatic, pictures of the transition state are most appropriate.9,11,12,43,81,82 For instance, weak coupling between spin states present in two-state reactivity could lead to significant nonadiabatic effects, but this possibility has not been thoroughly explored. Furthermore, it is not clear if nonadiabatic effects distinguish between $\sigma$ and $\pi$ reaction pathways for metal-oxo mediated $\mathrm{C}-\mathrm{H}$ activation. While these remain outstanding questions, there is also not yet sufficient experimental data on how general nonadiabatic effects are due to limited variable temperature KIE data. Much more work is needed in this area, both from experimentalists and theorists.

The experimental observation of nonadiabatic reactivity in a system which displays trends more consistent with an imbalanced transition state raises the question of how these two apparently paradoxical theoretical limits can both be viable in this case. Specifically, these results motivate the question of how off-diagonal thermodynamic parameters manifest in a rate theory which treats proton transfer quantum mechanically. As a starting point for interrogating this question, we hypothesized that vibronic coupling may serve as one possible mechanism.

\section{Imbalanced Transition State Effects Can Manifest Through Vibronic Coupling}

Nonadiabatic CPET can be conceptualized as occurring in two steps: (1) The reactants and the solvent reorganize to a configuration where the reactant and product have the same energy (Figure 4A), and (2) the electron and proton transfer from a discrete reactant vibronic state to a discrete
Table 1. Isotope dependence of Arrhenius parameters for various reactions.

\begin{tabular}{|c|c|c|c|}
\hline Reaction & Ref. & $\begin{array}{l}\mathrm{E}_{\mathrm{a}}(\mathrm{D})-\mathrm{E}_{\mathrm{a}}(\mathrm{H}) \\
(\mathrm{kcal} / \mathrm{mol})\end{array}$ & $\ln \left(A_{H} / A_{D}\right)$ \\
\hline CoO + DHA & g & $3.7(3)$ & $-3.3(5)$ \\
\hline \multirow{2}{*}{ CoO + Fluorene } & \multirow{2}{*}{$\mathrm{g}$} & $0.24(9)^{\mathrm{h}}$ & $0.9(2)^{h}$ \\
\hline & & $0.01(7)^{\mathrm{i}}$ & $0.1(1)^{\mathrm{i}}$ \\
\hline TAMLFeVO + EtPh & 64 & $3.3(8)$ & $-4(2)$ \\
\hline pipMe $\mathrm{LCu}^{\mathrm{III}} \mathrm{OH}+\mathrm{DHA}$ & 68 & $2.9(4)$ & $-3(2)$ \\
\hline $\mathrm{LCu}{ }^{\mathrm{III} O H}+\mathrm{DHA}$ & 68 & $0.4(2)$ & $3(3)$ \\
\hline${ }^{\mathrm{NO}} \mathrm{LCu}^{\mathrm{III}} \mathrm{OH}+\mathrm{DHA}$ & 68 & $3.6(3)$ & $-5.0(7)$ \\
\hline \multirow{2}{*}{$\left(\mathrm{L}^{\mathrm{iPr}}{ }_{3} \mathrm{Cu}^{\mathrm{III}}\right)_{2}(\mu-\mathrm{O})_{2}{ }^{2+a, b}$} & 67 & $0.5(7)$ & $2.0(7)$ \\
\hline & 66 & $1.9(7)$ & $-0.7(1)$ \\
\hline$\left(\mathrm{L}^{\mathrm{Bn}}{ }_{3} \mathrm{Cu}^{\mathrm{III}}\right)_{2}(\mu-\mathrm{O})_{2}^{2+\mathrm{a}}$ & 66 & $2.5(7)$ & $-1.6(1)$ \\
\hline$\left(\mathrm{Tp}^{\prime \prime} \mathrm{Co}^{\mathrm{II}}\right)_{2}\left(\mu-\mathrm{O}_{2}\right)^{\mathrm{a}}$ & 65 & $2.8(7)$ & $-2.0(7)$ \\
\hline $\begin{array}{c}\mathrm{Ru}^{\mathrm{III}}(\mathrm{bpy}) 3^{3+} / \mathrm{H}_{2} \mathrm{O}+ \\
\mathrm{PhOH}\end{array}$ & 62 & $1.68(4)$ & $-1.40(7)$ \\
\hline $\begin{array}{c}\text { 4-охо-ТЕМРО• + } \\
\text { ТЕМРОН }\end{array}$ & 71 & $0.3(6)$ & $1.1(6)$ \\
\hline 9a $H$-Quinolozined & 73 & $-0.01(2)$ & $1.64(3)$ \\
\hline SLOe & 79 & $0.9(2)$ & $2.9(3)$ \\
\hline PHM $^{f}$ & 80 & $0.4(3)$ & $1.8(5)$ \\
\hline
\end{tabular}

aActivation of a ligand $\mathrm{C}-\mathrm{H}$ bond. bTwo distinct sets of parameters are reported for this compound. cValues reported in DCM; similar results were also reported in MeCN. dSigmatropic rearrangement to $4 H$-Qunolozine. eSoybean Lipoxygenase- 1 fPeptidylglycine $\alpha$-hydroxylating monoxogyenase. gThis work. hPrimary KIE iSecondary KIE. Where necessary, reported changes in entropy in e.u. were converted to unitless $\ln \left(A_{H} / A_{D}\right)$ by multiplication of both value and error by 0.503 ; changes in enthalpy were used as reported for activation energy differences.

product vibronic state nonadiabatically (Figure 4B; the electron and proton are theoretically treated as two separate particles, even for concerted reactivity).4,83,84 The first step places the reactant and product vibronic wavefunctions at
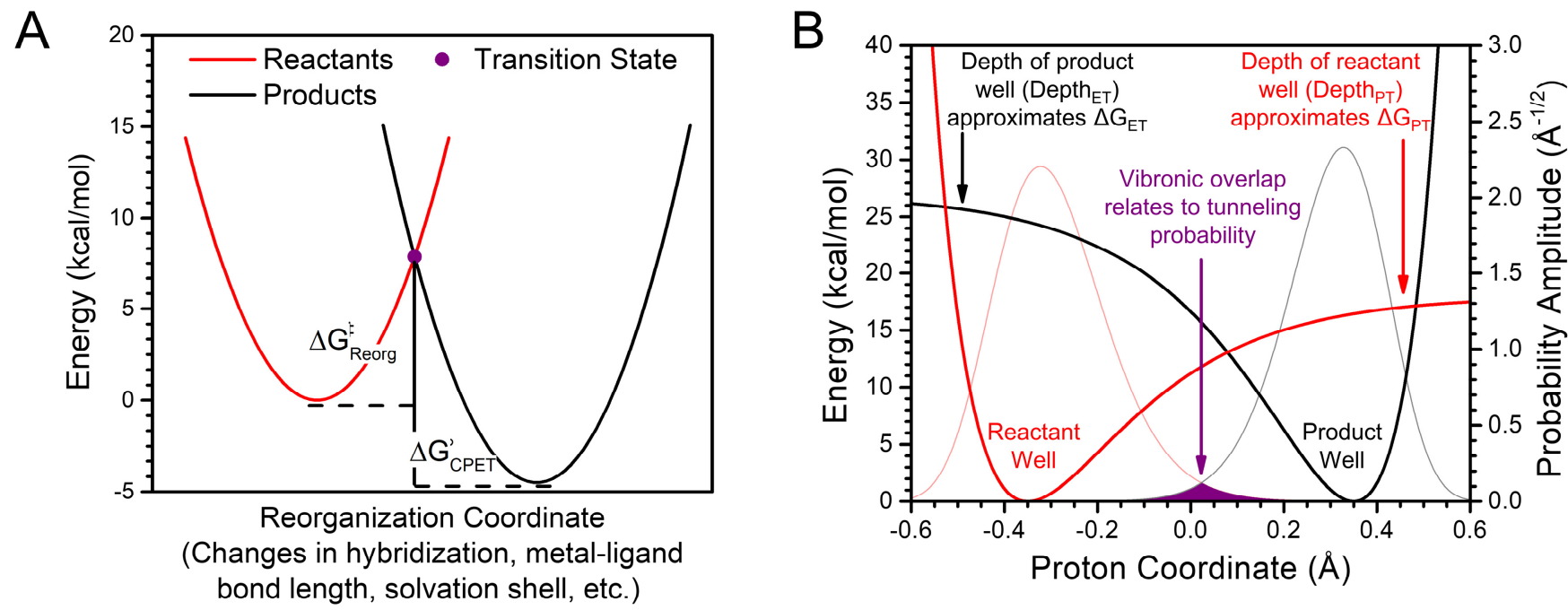

Figure 4. (A) Energy along the reorganization coordinate of CPET. (B) Energy along the proton coordinate at the reorganized transition state. The solid lines are the proton potential energy surfaces and the lighter lines represent the corresponding ground state vibronic wavefunctions of the proton. 
A

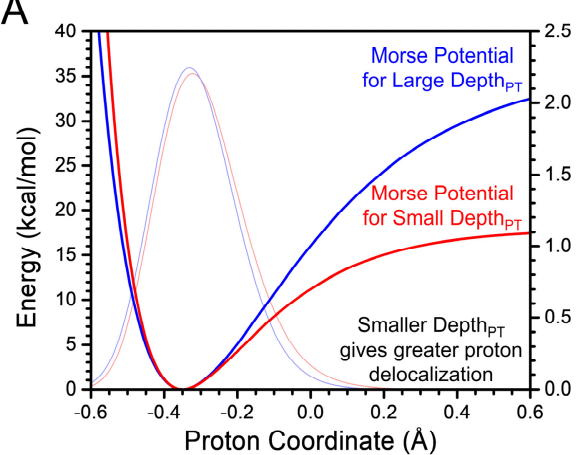

B

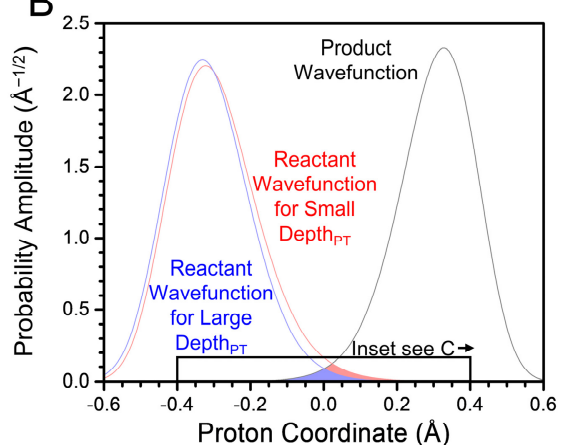

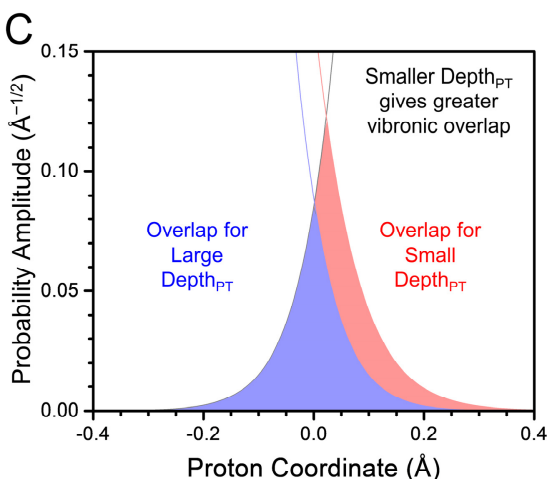

Figure 6. (A) Two reactant Morse potentials with varying depths (red and blue, left y-axis) with their ground state vibronic wavefunctions (light red and light blue, respectively, right y-axis). (B) The wavefunctions in (A) overlapping with a product vibronic wavefunction. Common overlap is show in in blue, with additional overlap of the shallower potential's wavefunction shown in red. (C) Zoomed version of the overlapping region in (B).

an equal energy, ensuring that energy is conserved when the transfer occurs. The second step, the actual electron and proton transfer itself, has a rate dependent on the coupling between the reactant and product vibronic states. Several formulations for this coupling exist, ${ }^{47,85-87}$ but it is commonly taken as the product of an electronic coupling and the Franck-Condon overlap between proton wavefunctions. ${ }^{7,40,88,89}$

Thus, instead of representing proton transfer with a single potential energy surface characterized by an activation barrier to climb, this model envisions two separate potential energy wells which represent the restoring force holding the proton on either the reactant or the product. The vibronic overlap of the proton's wavefunctions in these two wells, along with the with the electronic coupling between them, takes the place of the barrier height in determining the rate of proton transfer. Greater vibronic overlap leads to faster reactivity in a manner analogous to greater orbital overlap leading to stronger bonding.

Model systems for nonadiabatic CPET typically use either parabolic or Morse potentials which are parameterized with the stretching frequency and bond dissociation energy of the relevant $\mathrm{X}-\mathrm{H}$ bond.7.40,88,89 However, it has been noted these potential energy wells do not adequately capture the anharmonicity seen in DFT-calculated potential wells of specific systems. ${ }^{89-93}$ This increased anharmonicity results in qualitatively wider potential wells, with a shallower rise away from the minima.

We hypothesized that this additional anharmonicity of the potential energy well can be approximated with Morse potentials whose depths are determined not by the BDFE of the bond in question but by the vertical energies of proton transfer or of electron transfer - the energy of transferring only the proton or electron from reactant to product. These vertical energies with all other atoms frozen in the reorganized state, hereafter referred to as DepthPT and DepthET, are expected to correlate with the adiabatic values of $\Delta \mathrm{G}_{\mathrm{PT}}^{\circ}$

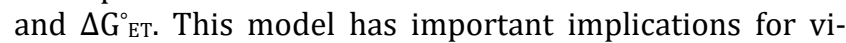
bronic overlap as shallower wells are wider and more anharmonic. This anharmonicity should lead to larger vibronic overlap and hence the possibility of higher rates.
This hypothesis therefore provides a mechanism by which a decrease in $\Delta \mathrm{G}_{\mathrm{PT}}^{\circ}$ and $\Delta \mathrm{G}_{\mathrm{ET}}^{\circ}$ can influence the reaction of CPET independent of concomitant decrease in

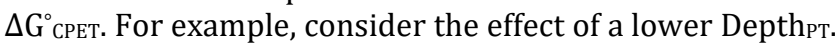
The lower potential energy away from the minima results in a more delocalized vibronic wavefunction which penetrates the barrier to a larger extent (Figure 6A, red curve). The overlap with a product vibronic state increases (Figure $6 \mathrm{~B}, \mathrm{C}$ ), and thus the overall reaction rate increases (Figure $5)$.

Importantly, this emergent picture aligns well with parallel models in semiclassical transition state theory. For instance, this overall model is reminiscent of structure-activity relationships in a More $0^{\prime}$ Ferral-Jencks plot: the lowering of $\Delta \mathrm{G}^{\circ}$ TT results in an increased probability of the proton being closer to the product, which in turn accelerates the reaction. ${ }^{34-36} \mathrm{We}$ also note that this model is complementary to models which invoke the changes to reorganization energy to account for reactivity trends; for example, HammesSchiffer and coworkers have found that for certain CPET reactions a more basic acceptor requires less energy to

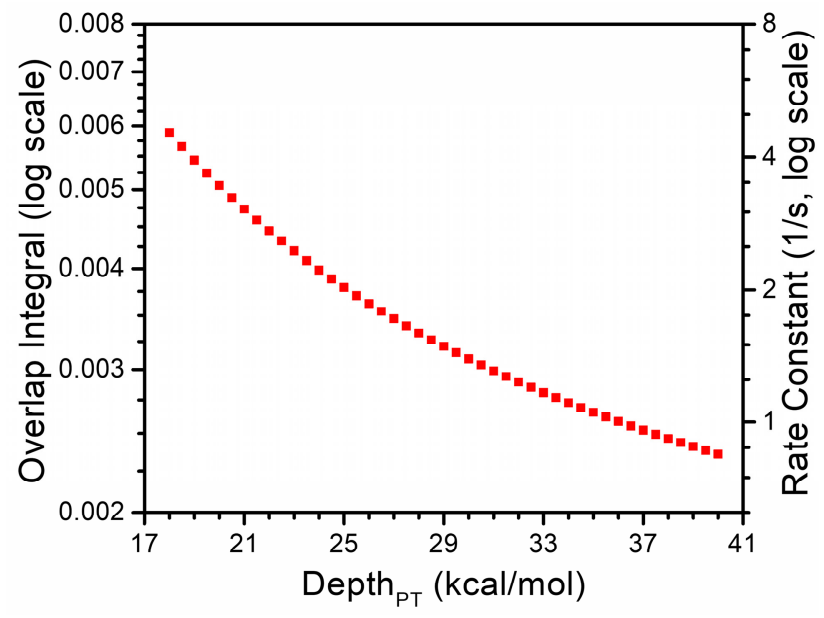

Figure 5. The overlap integral and simulated rate constant as a function of the depth of the (DepthPT). The product potential depth (DeptheT) is $27 \mathrm{kcal} / \mathrm{mol}$. See text for a description of the parameters used in the calculations. Both y-axes are logarithmic scales. 
A

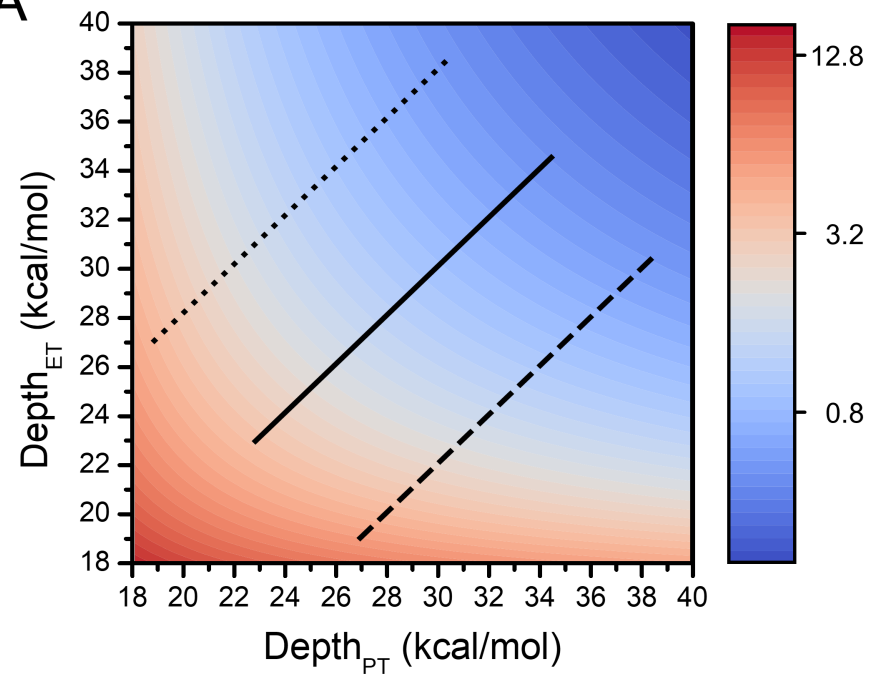

B

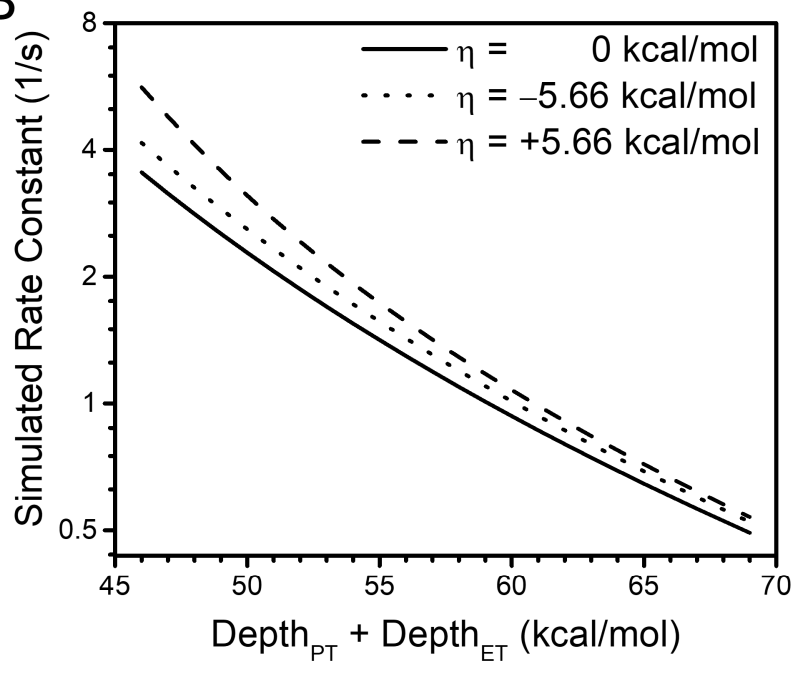

Figure 7. Simulated rate constants for CPET as a function of proton and electron transfer depth. (A) The effect of both DepthPT and Depth $_{\text {ET }}$ on the simulated rate constant $(1 / \mathrm{s})$ which is shown from blue to red according to the sidebar. The lines illustrate crosssections that are plotted in (B). (B) Plot of simulated rate constants versus DepthPT + DeptheT for DepthPT $>$ Depth $_{\mathrm{ET}}$ (dashed line), Depth $_{\mathrm{PT}}=$ Depth $_{\mathrm{ET}}$ (solid line), and Depth $\mathrm{PT}<$ Depth $_{\mathrm{ET}}$ (dotted line). Simulated rate constants are shown on a logarithmic scale.

achieve a necessary tunneling distance (which can be considered as part of the reorganization). ${ }^{48}$

\section{Simulations Support Trends with Off-Diagonal Thermody- namic Values}

The overarching conclusion from this simple model is that the depth of the potential wells can have a substantial effect on nonadiabatic CPET reactivity through vibronic coupling (Figure 7). Shallow potential energy wells, i.e. lower Depth PT $_{\text {and }}$ aepthet, result in improved overlap and faster rate constants. With this general conclusion in mind, we then performed simulations of CPET rate constants with this model to determine if some of the previously observed trends with off-diagonal thermodynamic effects can be successfully predicted. We assumed $\Delta \mathrm{G}_{\mathrm{CPET}}^{\circ}=-5 \mathrm{kcal} / \mathrm{mol}$, reorganization energy $\lambda=40 \mathrm{kcal} / \mathrm{mol}$, tunneling distance $\mathrm{R}=$ $0.7 \AA$, reactant $\mathrm{X}-\mathrm{H}$ stretching frequency $\omega=2900 \mathrm{~cm}^{-1}$, product X-H stretching frequency $\omega=3500 \mathrm{~cm}^{-1}, \mathrm{~T}=296 \mathrm{~K}$, and electronic coupling $\mathrm{V}_{\mathrm{el}}=100 \mathrm{~cm}^{-1}$ for these models (Figure 5 uses $\mathrm{V}_{\mathrm{el}}=106 \mathrm{~cm}^{-1}$ to better align the two $\mathrm{y}$-axes). These parameters are reasonable for CPET with the proton traversing from carbon to oxygen.7,14

The predominant effect that we observe from these simulations is a marked dependency on the combination of

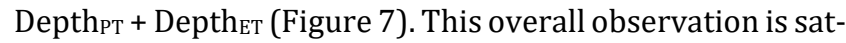
isfyingly consistent with a recent statistical analysis on the rates of reaction of a broad family of transition metal-oxo complexes from our group. ${ }^{94}$ Interestingly, however, the model provides a more nuanced picture of this trend. When

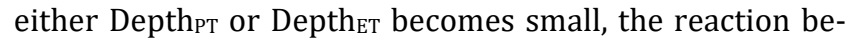
comes more sensitive to this lower depth, as illustrated by the decreased curvature of the upper-left and bottom right corners of Figure 7A. This more subtle trend goes further than our previous statistical analysis in that it explains the apparent insensitivity of the reactivity of $\mathbf{C o O}$ with $\Delta \mathrm{G}_{\mathrm{ET}}^{\circ}$ due to the particularly low values of $\Delta \mathrm{G}_{\mathrm{PT}}^{\circ}$ and comparatively high values of $\Delta \mathrm{G}_{\mathrm{ET}}^{\circ}$ in this system.
A consequence of the low sensitivity to Depthet at low

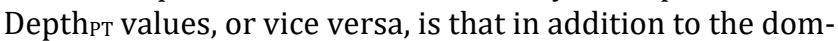
inant trend with DepthPT + Depth $_{\mathrm{ET}}$ there is also a trend with the asynchronicity parameter $\eta$ previously presented by Srnec and coworkers ( $\eta$ is proportional to DepthPT Depth $\left._{\text {ET }}\right) .^{9}$ Namely, our model predicts that as $\eta$ becomes larger rates should accelerate independent of changes in Depth $_{\mathrm{PT}}+$ Depth $_{\mathrm{ET}}$, in agreement with the previous analysis by Srnec and coworkers. Furthermore, as the variation in the difference in Depth PT $_{\text {and }}$ Depth ET $_{\mathrm{ET}}$ may be much larger than the variation in their sum, it is possible that this subtle effect may be dominant in some systems even though a more significant dependence on Depth ${ }_{\mathrm{PT}}+$ Depth $_{\mathrm{ET}}$ is predicted.

Intriguingly, there is also asymmetry in the dependence on $\eta$. The rate constant is less affected by more negative $\eta$

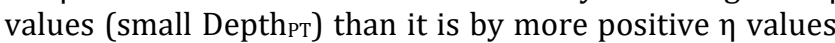
(small Deptheт); that is, systems with positive $\eta$ are expected to react more quickly than systems with negative $\eta$ (Figure 7B). This preference for low Depthet over low

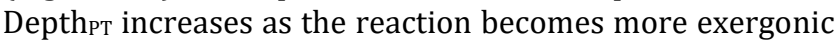
(See SI). However, if the reaction is made to be endergonic, the preference is reversed: a lower Depth ${ }_{\mathrm{PT}}$ (negative $\eta$ ) is more effective than a lower Depthet (positive $\eta$, see SI). We propose that this trend is due to the involvement of excited states in the reaction. Excited vibronic states are more dramatically affected by the decreasing well depths than the ground vibronic state (See SI). Exergonic reactions see a relatively large contribution of reactivity from the ground vibrational state into excited vibrational states, ${ }^{49}$ and therefore the greater sensitivity of excited vibronic states to the depth of the well translates into a greater sensitivity in DepthET. Conversely, excited reactant vibronic states are more important in endergonic reactions, and are more ac-

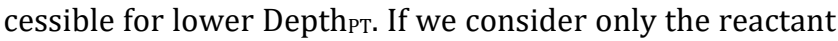
ground vibronic state to product ground vibronic state reactivity, we observe that while reactions with $|\eta|>0$ are still favorable, there is no longer an asymmetry between positive and negative $\eta$ (See SI). 
While the asymmetry in the dependence on $\eta$ is subtle, it may be of relevance to selectivity in $\mathrm{C}-\mathrm{H}$ activation reactions. For instance, compound I in cytochrome P450 performs uphill reactivity and metal-oxo complexes in P450 have been found to be quite basic. ${ }^{13,95,96}$ It is possible that a lower $\Delta \mathrm{G}_{\mathrm{PT}}^{\circ}$ is beneficial for selectivity in this system outside of its contribution to $\Delta \mathrm{G}^{\circ}$ СРET. Important roles for the high basicity of P450 enzymes have been invoked previously,13,95 but not in this particular context.

In short, this model, which uses comparatively simple Morse potentials to capture the effect of variable depths of proton potential energy surfaces, does a remarkably good job in predicting and matching experimental observations. Previous experimental trends in $\Delta \mathrm{G}_{\mathrm{PT}}^{\circ}$ and $\Delta \mathrm{G}_{\mathrm{ET}}^{\circ}$ which have been ascribed to imbalanced transition states are independently reproduced. Furthermore, this model also captures subtleties in these trends which fill in speculation or gaps from previous analyses. While the quantitative utility of this picture is limited, our results do convincingly demonstrate that reactivity trends ascribed to imbalanced transition states are not only compatible with nonadiabatic reactions, but also that these trends are a natural extension of vibronic overlap in these processes.

\section{Conclusions}

Based on the variable temperature KIEs reported herein, we find evidence of extensive tunneling in CPET reactions between $\mathbf{C o O}$ and $\mathrm{C}-\mathrm{H}$ bonds. For the reactivity with fluorene, temperature insensitive KIEs indicate tunneling which is so extensive that reactivity must proceed through a nonadiabatic process and cannot be explained with semiclassical theories of proton transfer. This is a clear demonstration that semiclassical structure-activity relationships are not sufficient to explain the CPET reactions of metal-oxygen complexes. Specifically, this result calls for a quantum mechanism for how off-main-diagonal free energies can affect nonadiabatic CPET reactions.

We introduce $\Delta \mathrm{G}_{\mathrm{PT}}^{\circ}$ and $\Delta \mathrm{G}_{\mathrm{ET}}^{\circ}$ into a nonadiabatic rate framework by approximating the reactant/product proton potential energy wells with Morse potentials whose depths correlate with $\Delta \mathrm{G}_{\mathrm{PT}}^{\circ} / \Delta \mathrm{G}_{\mathrm{ET}}^{\circ}$. This relatively simple model allows us to correlate rates with vibronic overlap which is directly dependent on this Morse potential depth. The source of this effect is increased penetration of the vibronic wavefunction across the proton coordinate when sole proton or electron transfer is more favorable, which is reminiscent of structure-activity relationships in semiclassical theory.

Simulations with this model demonstrate that off-maindiagonal free energies can have a substantial effect on the rate constants of nonadiabatic reactions. Simulated data reproduces experimental trends found in the sum of $\Delta \mathrm{G}_{\text {Рт }}^{\circ}$ and $\Delta \mathrm{G}^{\circ} \mathrm{ET}$ as well as previously reported dependencies on $\eta$, all within a nonadiabatic framework. Furthermore, this model not only supports previous studies, but it also makes apparent more subtle trends which help to explain previously speculative observations of rate dependencies and reactivity trends. Finally, these subtle trends also motivate deeper inspection of the importance of off-diagonal thermodynamic effects as a design principle.
These results reconcile the semiclassical predictions and explanations of asynchronous reactivity within a nonadiabatic context. Not only are semiclassical structure-activity relationships viable in a nonadiabatic framework, they are expected due to vibronic overlap arising from potential well anharmonicity. This observation both validates on a theoretical level the burgeoning field of imbalanced CPET reactivity, as well as motivates further study into how these effects dictate selectivity and reactivity. Furthermore, while this study has focused on the imbalanced transition state of a transition metal-oxo complex, we postulate that similar synergies between other semiclassical reactivity trends and nonadiabatic reactivity likely exist in CPET reactions. Asynchronous reactivity is far from the only theory regarding the rate of CPET, or of proton transfer more generally, which has been analyzed predominantly in a semiclassical context. Finally, we note that there is a paucity of experimental data regarding whether nonadiabatic transfer is the norm for metal-based oxidants. Resolving how broadly important nonadiabatic effects are in CPET reactions, and whether or not theories developed within a semiclassical framework hold in a nonadiabatic framework, is an important avenue for future research.

\section{Experimental Section}

Unless stated otherwise, all manipulations were performed under an inert atmosphere in MBraun gloveboxes or using standard Schlenk techniques. $\mathrm{PhB}\left({ }^{t} \mathrm{BuIm}\right)_{3} \mathrm{Co}^{\mathrm{III}} \mathrm{O}$ (CoO), $d_{1}$-fluorene, $d_{2}$-fluorene, and $d_{4}$-DHA were synthesized according to literature procedures; ${ }^{97-100}$ all other chemicals were obtained from commercial sources. We found that to selectively obtain $d_{1}$-fluorene and not $d_{2}$-fluorene it was critical to use exactly one equivalent of n-butyllithium and to rapidly quench with $\mathrm{D}_{2} \mathrm{O}$. For $d_{2}$-fluorene and $d_{4}$-DHA, we found using enough $d_{6}$-DMSO to fully dissolve the starting substrate allowed us to achieve higher isotopic purity but suspect heating the reaction only resulted in decreased yield. All substrates were recrystallized from hot hexanes prior to use. Rate constants were simulated using standard nonadiabatic formulas for CPET reactions, ${ }^{49,101}$ and formulas for the Morse potential energies and wavefunctions were taken from the literature. ${ }^{102}$ All data analysis was performed in python 3.7 utilizing Numy, Scipy, and Matplotlib packages. ${ }^{103-105}$

\section{Measurement of KIEs by Competition Experiments}

Competition experiments were run with $1.25 \mathrm{mM}$ of $\mathbf{C o O}$ and 20 equivalents of substrate in $2.0 \mathrm{~mL}$ of toluene. In a typical experiment, the substrate was dissolved in $1.9 \mathrm{~mL}$ of solvent and equilibrated to the desired temperature (a sealed vial in the glovebox freezer for $239 \mathrm{~K}$ data, a taped vial in an out of glovebox ice bath for $273 \mathrm{~K}$ data, a sealed vial set on the glovebox floor for $296 \mathrm{~K}$ data, and a sealed vial set in a glovebox heat block for $323 \mathrm{~K}$ data), then $100 \mu \mathrm{L}$ of $\mathbf{C o O}$ stock solution ( $25 \mathrm{mM}$ ) was added via syringe. The reaction was run to $>99 \%$ completion, as determined by five time constants (inverse rate constant) of the expected rate for the 10 equivalents of reactive $\mathrm{C}-\mathrm{H}$ bonds based on an Arrhenius analysis of the fluorene $d_{0}$-kinetic data (see below and SI). After the reaction, it was passed through either alumina or silica to remove Co containing products, dried, redissolved in DCM, and analyzed by GC-MS. 
GC-MS analysis was performed with an Agilent 7890B GC system and Agilent 4977A MSD. Chemical ionization was used to avoid fractionation. The amount of each isotopomer was determined by measuring a reference spectrum with no deuterium and fitting the partially deuterated spectra to a sum of the reference spectra shifted by each possible amount of deuterium; i.e. intensity at $\mathrm{m} / \mathrm{z}$ was modeled as inten $\frac{m}{z}=\sum r e f_{\frac{m}{z}-n} p_{n}$; with $r e f_{\frac{m}{z}-n}$ being the intensity of the reference spectra at $\mathrm{m} / \mathrm{z}-\mathrm{n}$ and $\mathrm{p}_{\mathrm{n}}$ giving the amount of $d_{\mathrm{n}}$-compound in the mixture; these $\mathrm{p}_{\mathrm{n}}$ were fit with least squares and then normalized to add to 1 . A weighted sum of the amount of deuterium in each isotopomer could be used for a total amount proportion of deuterium $p_{D}$ in each sample $\left(p_{D}=\sum p_{n} n\right)$. Fluorene was fit for $\mathrm{m} / \mathrm{z}=195-199$, DHA was fit to $\mathrm{m} / \mathrm{z}=209-215$, and bifluoreneyl fit to $\mathrm{m} / \mathrm{z}=331-$ 335. The Fluorene and DHA peaks correspond to ionization with $\mathrm{C}_{2} \mathrm{H}_{5}{ }^{+}$, which were used to avoid complications with hydride/deuteride loss which complicated the features for ionization with $\mathrm{H}^{+}$. No $\mathrm{M}-1$ peak was observed for bifluorenyl, so the more intense peak with $\mathrm{H}^{+}$ionization was used. The experimental data was modeled with the formula:

$$
\frac{p_{P H}}{p_{P D}}=\frac{2 k_{S H_{2}} p_{S H_{2}}+k_{S D H} p_{S H D}}{k_{S H D} p_{S D_{2}}+2 k_{S D_{2}} p_{S H D}}=\frac{2 K I E^{1{ }^{\circ}} K I E^{2^{\circ}} p_{S H_{2}}+K I E^{2^{\circ}} p_{S H D}}{K I E^{1^{\circ}} p_{S H D}+2 p_{S D_{2}}} \text { (1) }
$$

where the $p_{p}$ values indicate the proportions of protons/deuterons in the product, the $p_{S}$ values indicate the proportion of the indicated isotopomer in the substrate, and the $k_{\mathrm{s}}$ values indicate the rate of reaction with the indicated isotopomer $\left(k_{S H D}\right.$ refers to abstraction of an $\mathrm{H}$-atom with a secondary D-atom, and vice-versa for $k_{S D H}$ ). The KIEs were in turn modeled as

$$
K I E=\frac{A_{H}}{A_{D}} e_{a}^{\frac{E_{a}(D)-E_{a}(H)}{k T}}
$$

with the primary and secondary KIE having separate differences in activation energy $E_{a}(D)-E_{a}(A)$ and prefactor ratios $A_{H} / A_{D}$. Substituting equation (2) into equation (1) and performing a nonlinear least squares fit to the data yielded the reported values and errors of $E_{a}(D)-E_{a}(A)$ and $A_{H} / A_{D}$. We used mean values of the $p_{S}$ to prevent complicated correlations in error from biasing our best fit parameters; however, if we alter this mean value to be +/- the error in the $p_{S}$ it does not dramatically change the reported parameters. We see no evidence for scrambling between product radicals and the substrate in the GCMS peak of unreacted substrate and reported rate constants of $\mathrm{C}-\mathrm{H}$ scrambling are much smaller than reported dimerization rate constants. ${ }^{106,107}$

\section{Measurement of KIEs by Kinetic Experiments}

UV-vis data were collected with a Thermo Fisher EV0300 LS spectrometer and a Unisoku USP-203-B cryostat. All experiments were run with $1.25 \mathrm{mM}$ of $\mathbf{C o O}$ with 10 equivalents of substrate in $2 \mathrm{~mL}$ of toluene. In a typical experiment, a cuvette was prepared in an $\mathrm{N}_{2}$ glovebox with $1.8 \mathrm{~mL}$ toluene and $100 \mu \mathrm{L}$ of a stock solution of $250 \mathrm{mM}$ substrate, and then sealed with a screwtop cap with a puncturable septum. The cuvette was allowed to equilibrate to the desired temperature and then $100 \mu \mathrm{L}$ of $25 \mathrm{mM}$ CoO was injected via syringe. The absorbance at $470 \mathrm{~nm}$ was followed to $\sim 85 \%$ completion, and the rate constant $k_{\text {obs }}$ was obtained via a nonlinear least squares fit of the absorbance at $470 \mathrm{~nm}$ to $A(t)=A_{\Delta} e^{-k_{o b s} t}+A_{\infty}$.
For undeuterated substrates, $k_{\mathrm{H}}$ was estimated as the mean of three $k_{\text {obs }}$ measurements; the reported error is the standard error of the mean. Three $k_{\text {obs }}$ for deuterated substrate were similarly averaged to obtain $k_{\mathrm{D} \text {,obs }}$ and these were corrected with equation 3 for the actual extent of deuteration in the sample $\left(p_{\mathrm{D}}\right)$ to obtain $k_{\mathrm{D} \text {,corr }}$ values, which is what $k_{\mathrm{D}}$ refers to unless otherwise stated.

$$
k_{D, \text { corr }}=\frac{1}{p_{D}}\left(k_{D, o b s}-k_{H}\left(1-p_{D}\right)\right)(3)
$$

The $p_{\mathrm{D}}$ were determined both by ${ }^{1} \mathrm{H}$ NMR spectroscopy and GC-MS analysis, which closely agreed: For $d_{2}$-fluorene, ${ }^{1} \mathrm{H}$ NMR spectrum analysis gave $98 \%$ and GC-MS gave $96 \%$; $97 \%$ was used. For $d_{4}$-DHA, ${ }^{1} \mathrm{H}$-NMR spectrum analysis gave $>99 \%$ and GC-MS gave $95-97 \%$ (depending on how much of the $\mathrm{M}+29$ peak is assigned to a $d_{2}$ anthracene impurity); $98 \%$ was used. Error in the estimate of $p_{D}$ was not propagated because it would lead to complicated correlations between temperature data points which would confound later analysis (assuming this error is in the measurement and not in the sampling of the substrate). Instead, we confirmed that our conclusions are consistent within the whole range of possible $p_{D}$ values indicated above.

KIEs were then calculated as the ratio of $k_{\mathrm{H}}$ and $k_{\mathrm{D} \text {,corr, with }}$ error propagated from $k_{\mathrm{H}}$ and $k_{\mathrm{D} \text {,obs. Arrhenius parameters }}$ $E_{a}(D)-E_{a}(H)$ and $\ln \left(A_{H} / A_{D}\right)$ were calculated by a least squares linear fit of $\ln (\mathrm{KIE})$ estimates to $1 / \mathrm{kT}$. We also performed a nonlinear least squares fit of values of $E_{a}(D)$ $\mathrm{E}_{\mathrm{a}}(\mathrm{H})$ and $\ln \left(\mathrm{A}_{\mathrm{H}} / \mathrm{AD}_{\mathrm{D}}\right)$ to the experimental $k_{\mathrm{H}}$ and $k_{\mathrm{D} \text {,obs }}$ and found identical mean estimates and similar error bars.

For $d_{4}$-DHA at $263 \mathrm{~K}$, the rate was too slow to follow to completion; instead, we measured initial rates to $~ 9 \%$ completion, with the average $\mathrm{A}_{\Delta}$ of other temperatures used to convert the rate from of absorbance per second to a rate constant in per second $\left(k_{\mathrm{D}, \mathrm{obs}}=k_{\text {Init }} / \mathrm{A}_{\Delta}\right.$, with $k_{\text {Init }}$ the slope of the absorbance at $470 \mathrm{~nm}$ with time). For error propagation, the standard error of $\mathrm{A}_{\Delta}$ was used not the standard error of the mean, as variation in the value of $A_{\Delta}$. This data point is graphed to demonstrate consistency with the other data points, but not included in the linear fits to variable temperature. The rates for deuterated substrates were corrected by the proportion of hydrogens deuterated in the data, see the SI for a detailed delineation of how this was done and how error was handled. See the SI for a discussion of fluorene kinetic KIEs and a comparison with the competition KIEs.

\section{ASSOCIATED CONTENT}

Supporting Information. Comparison of competition and kinetic KIEs, graphs of kinetic and GC-MS data, analysis of penetration distances for ground and excited vibronic states, Morse rate color maps with varied parameters, and spreadsheets of simulated rate constants, and python scripts for calculating and visualizing simulated rate constants.

\section{AUTHOR INFORMATION}

\section{Corresponding Author}

*jsanderson@uchicago.edu

Notes

The authors declare no competing financial interests. 


\section{ACKNOWLEDGMENT}

This work was funded by the National Institutes of Health (R35 GM133470), the National Science Foundation (1654144), and the University of Chicago. J.E.S. thanks the Department of Defense for a National Defense Science and Engineering Graduate Fellowship (00003765) and J.S.A. thanks the Sloan Foundation for a Research Fellowship (FG-2019-11497). Rate constants were simulated using computational resources provided by the UChicago Research Computing Center.

\section{REFERENCES}

(1) Migliore, A.; Polizzi, N. F.; Therien, M. J.; Beratan, D. N. Biochemistry and Theory of Proton-Coupled Electron Transfer. Chem. Rev. 2014, 114 (7), 3381-3465.

(2) Dempsey, J. L.; Winkler, J. R.; Gray, H. B. Proton-Coupled Electron Flow in Protein Redox Machines. Chem. Rev. 2010, 110 (12), 7024-7039.

(3) Weinberg, D. R.; Gagliardi, C. J.; Hull, J. F.; Murphy, C. F.; Kent, C. A.; Westlake, B. C.; Paul, A.; Ess, D. H.; McCafferty, D. G.; Meyer, T. J. Proton-Coupled Electron Transfer. Chem. Rev. 2012, 112 (7), 4016-4093.

(4) Hammes-Schiffer, S. Proton-Coupled Electron Transfer: Moving Together and Charging Forward. J. Am. Chem. Soc. 2015 137 (28), 8860-8871.

(5) Siegbahn, P. E. M.; Blomberg, M. R. A. Quantum Chemical Studies of Proton-Coupled Electron Transfer in Metalloenzymes. Chem. Rev. 2010, 110 (12), 7040-7061.

(6) England, J.; Guo, Y.; Van Heuvelen, K. M.; Cranswick, M. A.; Rohde, G. T.; Bominaar, E. L.; Münck, E.; Que, L. A More Reactive Trigonal-Bipyramidal High-Spin Oxoiron(IV) Complex with a CisLabile Site. J. Am. Chem. Soc. 2011, 133 (31), 11880-11883.

(7) Edwards, S. J.; Soudackov, A. V.; Hammes-Schiffer, S. Analysis of Kinetic Isotope Effects for Proton-Coupled Electron Transfer Reactions. J. Phys. Chem. A 2009, 113 (10), 2117-2126.

(8) Sastri, C. V.; Lee, J.; Oh, K.; Lee, Y. J.; Lee, J.; Jackson, T. A.; Ray, K.; Hirao, H.; Shin, W.; Halfen, J. A.; Kim, J.; Que, L.; Shaik, S.; Nam, W. Axial Ligand Tuning of a Nonheme Iron(IV)-Oxo Unit for Hydrogen Atom Abstraction. Proc. Natl. Acad. Sci. U.S.A. 2007, 104 (49), 19181-19186.

(9) Bím, D.; Maldonado-Domínguez, M.; Rulíšek, L.; Srnec, M. Beyond the Classical Thermodynamic Contributions to Hydrogen Atom Abstraction Reactivity. Proc. Natl. Acad. Sci. U.S.A. 2018, 115 (44), E10287-E10294.

(10) Costentin, C. Electrochemical Approach to the Mechanistic Study of Proton-Coupled Electron Transfer. Chem. Rev. 2008, 108 (7), 2145-2179.

(11) Mandal, D.; Ramanan, R.; Usharani, D.; Janardanan, D.; Wang, B.; Shaik, S. How Does Tunneling Contribute to Counterintuitive H-Abstraction Reactivity of Nonheme Fe(IV)O Oxidants with Alkanes? J. Am. Chem. Soc. 2015, 137 (2), 722-733.

(12) Lai, W.; Li, C.; Chen, H.; Shaik, S. Hydrogen-Abstraction Reactivity Patterns from A to Y: The Valence Bond Way. Angew. Chem. Int. Ed. 2012, 51 (23), 5556-5578.

(13) Yosca, T. H.; Rittle, J.; Krest, C. M.; Onderko, E. L.; Silakov, A.; Calixto, J. C.; Behan, R. K.; Green, M. T. Iron(IV)Hydroxide PKa and the Role of Thiolate Ligation in $\mathrm{C}-\mathrm{H}$ Bond Activation by Cytochrome P450. Science 2013, 342 (6160), 825-829.

(14) Decornez, H.; Hammes-Schiffer, S. Model Proton-Coupled Electron Transfer Reactions in Solution: Predictions of Rates, Mechanisms, and Kinetic Isotope Effects. J. Phys. Chem. A 2000, 104 (41), 9370-9384.

(15) Wang, D.; Ray, K.; J. Collins, M.; R. Farquhar, E.; R. Frisch, J.; Gómez, L.; A. Jackson, T.; Kerscher, M.; Waleska, A.; Comba, P.; Costas, M.; Que, L. Nonheme Oxoiron(Iv) Complexes of Pentadentate N5 Ligands: Spectroscopy, Electrochemistry, and Oxidative Reactivity. Chem. Sci. 2013, 4 (1), 282-291.
(16) Tyburski, R.; Liu, T.; Glover, S. D.; Hammarström, L. Proton-Coupled Electron Transfer Guidelines, Fair and Square. J. Am. Chem. Soc. 2021, 143 (2), 560-576.

(17) S. Borovik, A. Role of Metal-Oxo Complexes in the Cleavage of C-H Bonds. Chem. Soc. Rev. 2011, 40 (4), 1870-1874.

(18) Warren, J. J.; Tronic, T. A.; Mayer, J. M. Thermochemistry of Proton-Coupled Electron Transfer Reagents and Its Implications. Chem. Rev. 2010, 110 (12), 6961-7001.

(19) Mayer, J. M. Understanding Hydrogen Atom Transfer: From Bond Strengths to Marcus Theory. Acc. Chem. Res. 2011, 44 (1), 36-46.

(20) Bell, R. P.; Hinshelwood, C. N. Acid Catalysis in Non-Aqueous Solvents. I.-The Rearrangement of N-Bromoacetanilide. Proc. R. Soc. A. 1934, 143 (849), 377-399.

(21) Evans, M. G.; Polanyi, M. Inertia and Driving Force of Chemical Reactions. Trans. Faraday Soc. 1938, 34 (0), 11-24.

(22) Mayer, J. M. Simple Marcus-Theory-Type Model for Hydrogen-Atom Transfer/Proton-Coupled Electron Transfer. J. Phys. Chem. Lett. 2011, 2 (12), 1481-1489.

(23) Darcy, J. W.; Kolmar, S. S.; Mayer, J. M. Transition State Asymmetry in C-H Bond Cleavage by Proton-Coupled Electron Transfer. J. Am. Chem. Soc. 2019, 141 (27), 10777-10787.

(24) Barman, S. K.; Jones, J. R.; Sun, C.; Hill, E. A.; Ziller, J. W.; Borovik, A. S. Regulating the Basicity of Metal-Oxido Complexes with a Single Hydrogen Bond and Its Effect on C-H Bond Cleavage. J. Am. Chem. Soc. 2019, 141 (28), 11142-11150.

(25) Goetz, M. K.; Anderson, J. S. Experimental Evidence for PKa-Driven Asynchronicity in C-H Activation by a Terminal Co(III)-Oxo Complex. J. Am. Chem. Soc. 2019, 141 (9), 4051-4062.

(26) Usharani, D.; Lacy, D. C.; Borovik, A. S.; Shaik, S. Dichotomous Hydrogen Atom Transfer vs Proton-Coupled Electron Transfer During Activation of X-H Bonds (X = C, N, O) by Nonheme IronOxo Complexes of Variable Basicity. J. Am. Chem. Soc. 2013, 135 (45), 17090-17104.

(27) Elwell, C. E.; Mandal, M.; Bouchey, C. J.; Que, L.; Cramer, C. J.; Tolman, W. B. Carboxylate Structural Effects on the Properties and Proton-Coupled Electron Transfer Reactivity of [CuO2CR]2+ Cores. Inorg. Chem. 2019, 58 (23), 15872-15879.

(28) Barman, S. K.; Yang, M.-Y.; Parsell, T. H.; Green, M. T.; Borovik, A. S. Semiempirical Method for Examining Asynchronicity in Metal-Oxido-Mediated C-H Bond Activation. Proc. Natl. Acad. Sci. U.S.A. 2021, 118 (36).

(29) Tedder, J. M. Which Factors Determine the Reactivity and Regioselectivity of Free Radical Substitution and Addition Reactions? Angew. Chem. Int. Ed. 1982, 21 (6), 401-410.

(30) Gentry, E. C.; Knowles, R. R. Synthetic Applications of Proton-Coupled Electron Transfer. Acc. Chem. Res. 2016, 49 (8), 1546-1556.

(31) Markle, T. F.; Darcy, J. W.; Mayer, J. M. A New Strategy to Efficiently Cleave and Form C-H Bonds Using Proton-Coupled Electron Transfer. Sci. Adv. 2018, 4 (7), eaat5776.

(32) Costentin, C.; Savéant, J.-M. Hydrogen and Proton Exchange at Carbon. Imbalanced Transition State and Mechanism Crossover. Chem. Sci. 2020, 11 (4), 1006-1010.

(33) Liu, T.; Tyburski, R.; Wang, S.; Fernández-Terán, R.; Ott, S.; Hammarström, L. Elucidating Proton-Coupled Electron Transfer Mechanisms of Metal Hydrides with Free Energy- and PressureDependent Kinetics. J. Am. Chem. Soc. 2019, 141 (43), 1724517259.

(34) Jencks, W. P. A Primer for the Bema Hapothle. An Empirical Approach to the Characterization of Changing Transition-State Structures. Chem. Rev. 1985, 85 (6), 511-527.

(35) Anslyn, E. V.; Dougherty, D. A. Modern Physical Organic Chemistry; University Science Books: Mill Valley, California, 2006.

(36) O'Ferrall, R. A. M. Relationships between E2 and E1cB Mechanisms of $\beta$-Elimination. J. Chem. Soc. B 1970, No. 0, 274-277.

(37) Bernasconi, C. F. The Principle of Imperfect Synchronization: I. Ionization of Carbon Acids. Tetrahedron 1985, 41 (16), 3219-3234. 
(38) Bernasconi, C. F. The Principle of Nonperfect Synchronization: Recent Developments. In Advances in Physical Organic Chemistry; Richard, J. P., Ed.; Academic Press, 2010; Vol. 44, pp 223-324.

(39) Bell, R. P. The Tunnel Effect in Chemistry; Chapman and Hall: London; New York, 1980.

(40) Klinman, J. P. A New Model for the Origin of Kinetic Hydrogen Isotope Effects. J. Phys. Org. Chem. 2010, 23 (7), 606-612.

(41) Layfield, J. P.; Hammes-Schiffer, S. Hydrogen Tunneling in Enzymes and Biomimetic Models. Chem. Rev. 2014, 114 (7), 3466-3494.

(42) Meisner, J.; Kästner, J. Atom Tunneling in Chemistry. Angew. Chem. Int. Ed. 2016, 55 (18), 5400-5413.

(43) Mandal, D.; Shaik, S. Interplay of Tunneling, Two-State Reactivity, and Bell-Evans-Polanyi Effects in C-H Activation by Nonheme Fe(IV)O Oxidants. J. Am. Chem. Soc. 2016, 138 (7), 20942097.

(44) Bae, S. H.; Li, X.-X.; Seo, M. S.; Lee, Y.-M.; Fukuzumi, S.; Nam, W. Tunneling Controls the Reaction Pathway in the Deformylation of Aldehydes by a Nonheme Iron(III)-Hydroperoxo Complex: Hydrogen Atom Abstraction versus Nucleophilic Addition. J. Am. Chem. Soc. 2019, 141 (19), 7675-7679.

(45) Maldonado-Domínguez, M.; Bím, D.; Fučík, R.; Čurík, R.; Srnec, M. Reactive Mode Composition Factor Analysis of Transition States: The Case of Coupled Electron-Proton Transfers. Phys. Chem. Chem. Phys. 2019, 21 (45), 24912-24918.

(46) Kiefer, P. M.; Hynes, J. T. Kinetic Isotope Effects for Nonadiabatic Proton Transfer Reactions in a Polar Environment. 1. Interpretation of Tunneling Kinetic Isotopic Effects. J. Phys. Chem. A 2004, 108 (52), 11793-11808.

(47) Hammes-Schiffer, S. Proton-Coupled Electron Transfer: Classification Scheme and Guide to Theoretical Methods. Energy Environ. Sci. 2012, 5 (7), 7696-7703.

(48) Sayfutyarova, E. R.; Lam, Y.-C.; Hammes-Schiffer, S. Strategies for Enhancing the Rate Constant of $\mathrm{C}-\mathrm{H}$ Bond Cleavage by Concerted Proton-Coupled Electron Transfer. J. Am. Chem. Soc. 2019, 141 (38), 15183-15189.

(49) Sayfutyarova, E. R.; Goldsmith, Z. K.; Hammes-Schiffer, S Theoretical Study of C-H Bond Cleavage via Concerted Proton-Coupled Electron Transfer in Fluorenyl-Benzoates. J. Am. Chem. Soc. 2018, 140 (46), 15641-15645.

(50) Bordwell, F. G. Equilibrium Acidities in Dimethyl Sulfoxide Solution. Acc. Chem. Res. 1988, 21 (12), 456-463.

(51) Luo, Y.-R. Handbook of Bond Dissociation Energies in Organic Compounds; CRC Press: Boca Raton, 2003.

(52) Bordwell, F. G.; Bares, J. E.; Bartmess, J. E.; McCollum, G. J.; Van der Puy, M.; Vanier, N. R.; Matthews, W. S. Carbon Acids. 12. Acidifying Effects of Phenyl Substituents.J. Org. Chem. 1977, 42 (2), 321-325.

(53) Zhang, X.-M.; Bordwell, F. G. Homolytic Bond Dissociation Energies of the Benzylic Carbon-Hydrogen Bonds in Radical Anions and Radical Cations Derived from Fluorenes, Triphenylmethanes, and Related Compounds. J. Am. Chem. Soc. 1992, 114 (25), 9787-9792.

(54) Bordwell, F. G.; Drucker, G. E.; McCollum, G. J. Stabilization of Carbanions by Polarization of Alkyl Groups on Nonadjacent Atoms. J. Org. Chem. 1982, 47 (13), 2504-2510.

(55) Bordwell, F. G.; Harrelson Jr, J. A. Acidities and Homolytic Bond Dissociation Energies of the $\mathrm{AC}-\mathrm{H}$ Bonds in Ketones in DMSO. Can. J. Chem. 1990, 68 (10), 1714-1718.

(56) Klinman, J. P.; Offenbacher, A. R. Understanding Biological Hydrogen Transfer Through the Lens of Temperature Dependent Kinetic Isotope Effects. Acc. Chem. Res. 2018, 51 (9), 19661974.

(57) Kohen, A.; Klinman, J. P. Enzyme Catalysis: Beyond Classical Paradigms. Acc. Chem. Res. 1998, 31 (7), 397-404.

(58) Schneider, M. E.; Stern, M. J. Arrhenius Preexponential Factors for Primary Hydrogen Kinetic Isotope Effects. J. Am. Chem. Soc. 1972, 94 (5), 1517-1522.
(59) Bonin, J.; Costentin, C.; Robert, M.; Savéant, J.-M.; Tard, C. Hydrogen-Bond Relays in Concerted Proton-Electron Transfers. Acc. Chem. Res. 2012, 45 (3), 372-381.

(60) Bonin, J.; Costentin, C.; Robert, M.; Savéant, J.-M. Pyridine as Proton Acceptor in the Concerted Proton Electron Transfer Oxidation of Phenol. Org. Biomol. Chem. 2011, 9 (11), 4064-4069.

(61) Sjödin, M.; Ghanem, R.; Polivka, T.; Pan, J.; Styring, S.; Sun, L.; Sundström, V.; Hammarström, L. Tuning Proton Coupled Electron Transfer from Tyrosine: A Competition between Concerted and Step-Wise Mechanisms. Phys. Chem. Chem. Phys. 2004, 6 (20), 4851-4858.

(62) Bonin, J.; Costentin, C.; Louault, C.; Robert, M.; Savéant, J.M. Water (in Water) as an Intrinsically Efficient Proton Acceptor in Concerted Proton Electron Transfers. J. Am. Chem. Soc. 2011, 133 (17), 6668-6674.

(63) Mahapatra, S.; Halfen, J. A.; Wilkinson, E. C.; Pan, G.; Cramer, C. J.; Que, L. Jr.; Tolman, W. B. A New Intermediate in Copper Dioxygen Chemistry: Breaking the 0-0 Bond To Form a \{Cu2(.Mu.-0)2\}2+ Core. J. Am. Chem. Soc. 1995, 117 (34), 88658866.

(64) Kundu, S.; Thompson, J. V. K.; Shen, L. Q.; Mills, M. R.; Bominaar, E. L.; Ryabov, A. D.; Collins, T. J. Activation Parameters as Mechanistic Probes in the TAML Iron(V)-Oxo Oxidations of Hydrocarbons. Chem. Eur. J. 2015, 21 (4), 1803-1810.

(65) Reinaud, O. M.; Theopold, K. H. Hydrogen Tunneling in the Activation of Dioxygen by a Tris(Pyrazolyl)Borate Cobalt Complex. J. Am. Chem. Soc. 1994, 116 (15), 6979-6980.

(66) Mahapatra, S.; Halfen, J. A.; Tolman, W. B. Mechanistic Study of the Oxidative N-Dealkylation Reactions of Bis( $\mu$-Oxo)Dicopper Complexes. J. Am. Chem. Soc. 1996, 118 (46), 11575-11586.

(67) Mahapatra, S.; Halfen, J. A.; Wilkinson, E. C.; Que, L.; Tolman, W. B. Modeling Copper-Dioxygen Reactivity in Proteins: Aliphatic C-H Bond Activation by a New Dicopper(II)-Peroxo Complex. J. Am. Chem. Soc. 1994, 116 (21), 9785-9786.

(68) Dhar, D.; Yee, G. M.; Spaeth, A. D.; Boyce, D. W.; Zhang, H.; Dereli, B.; Cramer, C. J.; Tolman, W. B. Perturbing the Copper(III)Hydroxide Unit through Ligand Structural Variation. J. Am. Chem. Soc. 2016, 138 (1), 356-368.

(69) Caldin, E. F.; Mateo, S. Kinetic Isotope Effects and Tunnelling in the Proton-Transfer Reaction between 4-Nitrophenylnitromethane and Tetramethylguanidine in Various Aprotic Solvents. J. Chem. Soc., Faraday Trans. 1 1975, 71 (0), 1876-1904.

(70) Giagou, T.; Meyer, M. P. Mechanism of the Swern Oxidation: Significant Deviations from Transition State Theory. J. Org. Chem. 2010, 75 (23), 8088-8099.

(71) Wu, A.; Mader, E. A.; Datta, A.; Hrovat, D. A.; Borden, W. T.; Mayer, J. M. Nitroxyl Radical Plus Hydroxylamine Pseudo SelfExchange Reactions: Tunneling in Hydrogen Atom Transfer. J. Am. Chem. Soc. 2009, 131 (33), 11985-11997.

(72) Kwart, H.; Benko, D. A.; Bromberg, M. E. Geometry of Transition States Involving Allylic Hydrogen Abstraction Elucidated by Isotope Effect Studies. J. Am. Chem. Soc. 1978, 100 (22), 7093-7094.

(73) Kwart, H.; Brechbiel, M. W.; Acheson, R. M.; Ward, D. C Observations on the Geometry of Hydrogen Transfer in [1,5] Sigmatropic Rearrangements. J. Am. Chem. Soc. 1982, 104 (17), 46714672.

(74) Kwart, H.; Brechbiel, M. W. Transition-State Geometry in the Ene Reactions of Mesoxalic Esters. J. Org. Chem. 1982, 47 (17) $3353-3355$

(75) Kwart, H.; George, T. J.; Louw, R.; Ultee, W. TransitionState Structures in Sulfoxide and Amine Oxide Thermolysis. J. Am. Chem. Soc. 1978, 100 (12), 3927-3928.

(76) Yoder, J. C.; Roth, J. P.; Gussenhoven, E. M.; Larsen, A. S. Mayer, J. M. Electron and Hydrogen-Atom Self-Exchange Reactions of Iron and Cobalt Coordination Complexes. J. Am. Chem. Soc. 2003, 125 (9), 2629-2640.

(77) Wu, A.; Mayer, J. M. Hydrogen Atom Transfer Reactions of a Ruthenium Imidazole Complex: Hydrogen Tunneling and the 
Applicability of the Marcus Cross Relation. J. Am. Chem. Soc. 2008, 130 (44), 14745-14754.

(78) Fukuzumi, S.; Kobayashi, T.; Suenobu, T. Unusually Large Tunneling Effect on Highly Efficient Generation of Hydrogen and Hydrogen Isotopes in PH-Selective Decomposition of Formic Acid Catalyzed by a Heterodinuclear Iridium-Ruthenium Complex in Water. J. Am. Chem. Soc. 2010, 132 (5), 1496-1497.

(79) Meyer, M. P.; Tomchick, D. R.; Klinman, J. P. Enzyme Structure and Dynamics Affect Hydrogen Tunneling: The Impact of a Remote Side Chain (I553) in Soybean Lipoxygenase-1. Proc. Natl. Acad. Sci. U.S.A. 2008, 105 (4), 1146-1151.

(80) Francisco, W. A.; Knapp, M. J.; Blackburn, N. J.; Klinman, J. P. Hydrogen Tunneling in Peptidylglycine $\alpha$-Hydroxylating Monooxygenase. J. Am. Chem. Soc. 2002, 124 (28), 8194-8195.

(81) Cho, K.-B.; Kim, E. J.; Seo, M. S.; Shaik, S.; Nam, W. Correlating DFT-Calculated Energy Barriers to Experiments in Nonheme Octahedral FeIVO Species. Chem. Eur. J. 2012, 18 (33), 1044410453.

(82) Mandal, D.; Mallick, D.; Shaik, S. Kinetic Isotope Effect Determination Probes the Spin of the Transition State, Its Stereochemistry, and Its Ligand Sphere in Hydrogen Abstraction Reactions of Oxoiron(IV) Complexes. Acc. Chem. Res. 2018, 51 (1), 107117.

(83) Marcus, R. A. On the Theory of Oxidation-Reduction Reactions Involving Electron Transfer. I. J. Chem. Phys. 1956, 24 (5), 966-978.

(84) Marcus, R. A.; Sutin, N. Electron Transfers in Chemistry and Biology. Biochim. Biophys. Acta, Rev. Bioenerg. 1985, 811 (3), 265-322.

(85) Georgievskii, Y.; Stuchebrukhov, A. A. Concerted Electron and Proton Transfer: Transition from Nonadiabatic to Adiabatic Proton Tunneling. J. Chem. Phys. 2000, 113 (23), 10438-10450.

(86) Skone, J. H.; Soudackov, A. V.; Hammes-Schiffer, S. Calculation of Vibronic Couplings for Phenoxyl/Phenol and Benzyl/Toluene Self-Exchange Reactions: Implications for Proton-Coupled Electron Transfer Mechanisms. J. Am. Chem. Soc. 2006, 128 (51), 16655-16663.

(87) Kuznetsov, A. M.; Ulstrup, J. Proton and Hydrogen Atom Tunnelling in Hydrolytic and Redox Enzyme Catalysis. Can. J. Chem. 1999, 77 (5-6), 1085-1096.

(88) Knapp, M. J.; Rickert, K.; Klinman, J. P. Temperature-Dependent Isotope Effects in Soybean Lipoxygenase-1: Correlating Hydrogen Tunneling with Protein Dynamics. J. Am. Chem. Soc. 2002, 124 (15), 3865-3874.

(89) Markle, T. F.; Zhang, M.-T.; Santoni, M.-P.; Johannissen, L. 0.; Hammarström, L. Proton-Coupled Electron Transfer in a Series of Ruthenium-Linked Tyrosines with Internal Bases: Evaluation of a Tunneling Model for Experimental Temperature-Dependent Kinetics. J. Phys. Chem. B 2016, 120 (35), 9308-9321.

(90) Markle, T. F.; Mayer, J. M. Concerted Proton-Electron Transfer in Pyridylphenols: The Importance of the Hydrogen Bond. Angewandte Chemie International Edition 2008, 47 (4), 738-740.

(91) Markle, T. F.; Rhile, I. J.; Mayer, J. M. Kinetic Effects of Increased Proton Transfer Distance on Proton-Coupled Oxidations of Phenol-Amines. J. Am. Chem. Soc. 2011, 133 (43), 17341-17352.

(92) Markle, T. F.; Tenderholt, A. L.; Mayer, J. M. Probing Quantum and Dynamic Effects in Concerted Proton-Electron Transfer Reactions of Phenol-Base Compounds. J. Phys. Chem. B 2012, 116 (1), 571-584.
(93) Liu, Y.; Liu, H.; Song, K.; Xu, Y.; Shi, Q. Theoretical Study of Proton Coupled Electron Transfer Reactions: The Effect of Hydrogen Bond Bending Motion. J. Phys. Chem. B 2015, 119 (25), 81048114.

(94) Schneider, J. E.; Goetz, M. K.; Anderson, J. S. Statistical Analysis of $\mathrm{C}-\mathrm{H}$ Activation by Oxo Complexes Supports Diverse Thermodynamic Control over Reactivity. Chem. Sci. 2021, 12 (11), 4173-4183.

(95) Green, M. T.; Dawson, J. H.; Gray, H. B. Oxoiron(IV) in Chloroperoxidase Compound II Is Basic: Implications for P450 Chemistry. Science 2004, 304 (5677), 1653-1656.

(96) Rittle, J.; Green, M. T. Cytochrome P450 Compound I: Capture, Characterization, and C-H Bond Activation Kinetics. Science 2010, 330 (6006), 933-937.

(97) Goetz, M. K.; Hill, E. A.; Filatov, A. S.; Anderson, J. S. Isolation of a Terminal Co(III)-Oxo Complex. J. Am. Chem. Soc. 2018, 140 (41), 13176-13180.

(98) Goldsmith, C. R.; Jonas, R. T.; Stack, T. D. P. C-H Bond Activation by a Ferric Methoxide Complex: Modeling the Rate-Determining Step in the Mechanism of Lipoxygenase. J. Am. Chem. Soc. 2002, 124 (1), 83-96.

(99) Bailey, R. J.; Card, P.; Shechter, H. Chemistry of 8-Substituted 1-Naphthylmethylenes and 2-Substituted Benzylidenes. A Simple Entry to $1 \mathrm{H}$-Cyclobuta[de]Naphthalenes. J. Am. Chem. Soc. 1983, 105 (19), 6096-6103.

(100) Brevard, Ch.; Kintzinger, J. P.; Lehn, J. M. Molecular dynamics-VI: Deuterium quadrupolar microdynamical labelling. Tetrahedron 1972, 28 (9), 2429-2445.

(101) Hammes-Schiffer, S.; Stuchebrukhov, A. A. Theory of Coupled Electron and Proton Transfer Reactions. Chem. Rev. 2010, 110 (12), 6939-6960.

(102) Dahl, J. P.; Springborg, M. The Morse Oscillator in Position Space, Momentum Space, and Phase Space. J. Chem. Phys. 1988, 88 (7), 4535-4547.

(103) Hunter, J. D. Matplotlib: A 2D Graphics Environment. Comput. Sci. Eng. 2007, 9 (3), 90-95.

(104) Virtanen, P.; Gommers, R.; Oliphant, T. E.; Haberland, M.; Reddy, T.; Cournapeau, D.; Burovski, E.; Peterson, P.; Weckesser, W.; Bright, J.; van der Walt, S. J.; Brett, M.; Wilson, J.; Millman, K. J.; Mayorov, N.; Nelson, A. R. J.; Jones, E.; Kern, R.; Larson, E.; Carey, C. J.; Polat, İ.; Feng, Y.; Moore, E. W.; VanderPlas, J.; Laxalde, D.; Perktold, J.; Cimrman, R.; Henriksen, I.; Quintero, E. A.; Harris, C. R.; Archibald, A. M.; Ribeiro, A. H.; Pedregosa, F.; van Mulbregt, P. SciPy 1.0: Fundamental Algorithms for Scientific Computing in Python. Nat. Methods 2020, 17 (3), 261-272.

(105) van der Walt, S.; Colbert, S. C.; Varoquaux, G. The NumPy Array: A Structure for Efficient Numerical Computation. Comput. Sci. Eng. 2011, 13 (2), 22-30.

(106) Warren, J. J.; Mayer, J. M. Predicting Organic Hydrogen Atom Transfer Rate Constants Using the Marcus Cross Relation. Proc. Natl. Acad. Sci. U.S.A. 2010, 107 (12), 5282-5287.

(107) Arends, I. W. C. E.; Mulder, P.; Clark, K. B.; Wayner, D. D. M. Rate Constants for Termination and TEMPO Trapping of Some Resonance Stabilized Hydroaromatic Radicals in the Liquid Phase. J. Phys. Chem. 1995, 99 (20), 8182-8189. 